\title{
Voltage Stability Control of Electrical Network Using Intelligent Load Shedding Strategy Based on Fuzzy Logic
}

\author{
Houda Jouini, Kamel Jemai, and Souad Chebbi \\ Electrical Engineering Department, Higher School of Sciences and Techniques of Tunis (ESSTT), \\ University of Tunis, 5 Avenue Taha Hussein, Montfleury 1008, Tunisia \\ Correspondence should be addressed to Kamel Jemai, kamel.jema@yahoo.fr
}

Received 28 June 2010; Accepted 9 December 2010

Academic Editor: Fernando Lobo Pereira

Copyright ( $) 2010$ Houda Jouini et al. This is an open access article distributed under the Creative Commons Attribution License, which permits unrestricted use, distribution, and reproduction in any medium, provided the original work is properly cited.

\begin{abstract}
As a perspective to ensure the power system stability and to avoid the vulnerability leading to the blackouts, several preventive and curative means are adopted. In order to avoid the voltage collapse, load shedding schemes represent a suitable action to maintain the power system service quality and to control its vulnerability. In this paper, we try to propose an intelligent load shedding strategy as a new approach based on fuzzy controllers. This strategy was founded on the calculation of generated power sensitivity degree related to those injected at different network buses. During the fault phase, fuzzy controller algorithms generate monitor vectors ensuring a precalculated load shedding ratio in the purpose to reestablish the power balance and conduct the network to a new steady state.
\end{abstract}

\section{Introduction}

Various disturbances occur in electrical networks every year which lead to blackouts. As the frequency and voltage represent two important parameters to the power system safety, it should have a continuous control of these parameters and this to ensure the best service quality. It is characterized by standard criteria related to the service continuity, the voltage profile, the purity of injected frequency, and the network static and transient robustness according to a set of possible exploitations and disturbance scenarios. The network vulnerability control is an important rivalry, since preventive and curative means can be considered in order to guarantee network service quality. In the case of vulnerable cascading events leading to blackouts, the load shedding will be the most desirable action avoiding network instability [1]. Different methods were proposed in order to decide the place and the quantity of loads to be shed. Indeed, Faranda et al. proposed a new load 
shedding approach called distributed interruptible load shedding [2]. Subramanian made a new model based on the sensitivity in the electric networks in conjunction with the linear programming for solutions of load shedding [3]. Tomšič et al. started with a dynamic model of the frequency that permits to simulate the impact of the most important system on the response of the frequency following disruptions and to determine the optimal number of load shedding stages and the percentage to shed in every stage [4]. Parker et al. used the medium-term dynamic simulation to prove the impact of the load shedding action and this in order to affect the appropriate systems control. We must to indicate that it's a voltage modal analysis combined with the determination of the reactive power margin [5]. Haidar et al. have a combined approach to basis of fuzzy logic and neurons networks to determine the percentage to shed to avoid the voltage collapse. It is a technique of electric system vulnerability control [6].

Girgis and Mathure developed an application based on the active power sensitivity for the variations of the frequency and voltage [7] and this to avoid the blackouts. Udupa et al. have developed an application of alleviation overload based on the fuzzy logic and neurons networks. This application consists in controlling sensitivity factors with the use of a load flow operational model [8]. S. J. Huang and C. C. Huang used a load shedding adaptive method with time for the electric system insulation [9]. Jouini et al. developed an application of load shedding for the Tunisian electric system based on the frequency observation while introducing the mixed criteria of frequency method [10].

All the authors who treat the sensitivity do not explain the influence of the power injected at each bus on the behavior of machines and consequently on the voltage level. The load shedding decision made in the previous works is only operated after a delayed analysis of consequences generated by the occurred faults. We develop, in this paper, within context, a new load shedding strategy based on the fuzzy logic named intelligent load shedding. Our motivation is the insurance of the electrical network continuity and avoids the collapse phenomenon. Thus, with this new strategy, we avoid some socioeconomic problems.

\section{Intelligent Load Shedding Strategy}

Disturbances leading to the voltage collapse phenomenon have stochastic characters. In a given bus, if the production source does not succeed in compensating the VAR reactive power, voltage level drops. The injected powers at bus $i$ are expressed as follows:

$$
\begin{aligned}
& P_{i}=\sum_{j=1}^{j=N_{\text {bus }}} Y_{i j} \cdot V_{i} \cdot V_{j} \cdot \cos \left(\alpha_{i}-\alpha_{j}-\theta_{i j}\right), \\
& Q_{i}=\sum_{j=1}^{j=N_{\text {bus }}} Y_{i j} \cdot V_{i} \cdot V_{j} \cdot \sin \left(\alpha_{i}-\alpha_{j}-\theta_{i j}\right),
\end{aligned}
$$

where $Y_{i j}, \theta_{i j}$ are the module and argument of the line admittance $i j, V_{i}, \alpha_{i}$ are the module and argument of voltage at bus $i, V_{j}, \alpha_{j}$ are the module and argument of voltage at bus $j$, and $N_{\text {bus }}$ is the number of studied network buses. 
The machine connected at bus $i$ must provide active and reactive powers, respectively, $P_{e i}, Q_{e i}$, where

$$
\begin{aligned}
P_{e i} & =P_{c i}+P_{i}, \\
Q_{e i} & =Q_{c i}+Q_{i},
\end{aligned}
$$

$P_{c i}$ and $Q_{c i}$ are, respectively, active and reactive powers consumed at bus $i$. follows:

Knowing that active and reactive powers injected at bus $i, P_{i}$, and $Q_{i}$ are expressed as

$$
\begin{gathered}
P_{i}=Y_{i i} \cdot V_{i}^{2} \cdot \cos \left(\theta_{i i}\right)+\sum_{j=1}^{j=N_{c i}} Y_{i j} \cdot V_{i} \cdot V_{j} \cos \left(\alpha_{i}-\alpha_{j}-\theta_{i j}\right), \\
Q_{i}=-Y_{i i} \cdot V_{i}^{2} \cdot \sin \left(\theta_{i i}\right)+\sum_{j=1}^{j=N_{c i}} Y_{i j} \cdot V_{i} \cdot V_{j} \sin \left(\alpha_{i}-\alpha_{j}-\theta_{i j}\right),
\end{gathered}
$$

where $Y_{i i}, \theta_{i i}$ are the module and argument of admittance at bus $i$.

In order to evaluate the influence of the consumed power level at different buses on the generated power level, we expressed the voltage $V_{j}$ at each bus, in the relation (2.1), according to the injected power $P_{j}$ as follows:

$$
V_{j}=\frac{P_{j}-\sum_{k=l}^{k=N_{\text {bus }}} Y_{j k} \cdot V_{j} \cdot V_{k} \cdot \cos \left(\alpha_{j}-\alpha_{k}-\theta_{j k}\right)}{Y_{j l} \cdot V_{l} \cdot \cos \left(\alpha_{j}-\alpha_{l}-\theta_{j l}\right)}, \quad(l \neq i) .
$$

By substitution of the voltage expression $V_{j}$ in the relation (2.1), we obtain

$$
\begin{aligned}
& P_{i}=\sum_{j=1}^{j=N_{\text {bus }}} Y_{i j} \cdot V_{i} \cdot \frac{P_{j}-\sum_{k=l}^{k=N_{\text {bus }}} Y_{j k} \cdot V_{j} \cdot V_{k} \cdot \cos \left(\alpha_{j}-\alpha_{k}-\theta_{j k}\right)}{Y_{j l} \cdot V_{l} \cdot \cos \left(\alpha_{j}-\alpha_{l}-\theta_{j l}\right)} \cdot \cos \left(\alpha_{i}-\alpha_{j}-\theta_{i j}\right), \\
& Q_{i}=\sum_{j=1}^{j=N_{\text {bus }}} Y_{i j} \cdot V_{i} \cdot \frac{P_{j}-\sum_{k=l}^{k=N_{\text {bus }}} Y_{j k} \cdot V_{j} \cdot V_{k} \cdot \cos \left(\alpha_{j}-\alpha_{k}-\theta_{j k}\right)}{Y_{j l} \cdot V_{l} \cdot \cos \left(\alpha_{j}-\alpha_{l}-\theta_{j l}\right)} \cdot \sin \left(\alpha_{i}-\alpha_{j}-\theta_{i j}\right) .
\end{aligned}
$$

By using (2.5), we succeed in expressing the generated power according to a generalized form, taking into account the injected powers in the network weighed by multipliers coefficients $C_{p d j}$ in order to have

$$
P_{e i}=P_{c i}+\sum_{\substack{j=1 \\ j \neq i}}^{j=N_{\text {bus }}} C_{p d j} \cdot P_{j}
$$

The coefficients $C_{p d j}$ materialize the influence of injected powers at different buses on the machine stability. These same coefficients depend on the consumed power levels in the network. 
During the failure phase, the generated power level depends on fluctuations affecting injected powers at each bus. In order to evaluate the machine sensitivity range to injected powers at each bus, we linearized the expression of the generated power. We developed a mathematical model in order to estimate the influence of the injected power level at each bus on the dynamic behavior of each machine. From the relation (2.6), we established the matrix model describing the linearization:

$$
\left[\begin{array}{c}
\Delta P_{e 1} \\
\Delta P_{e 2} \\
\vdots \\
\Delta P_{e n}
\end{array}\right]=\left[\begin{array}{llll}
\frac{\partial P_{e 1}}{\partial P_{1}} & \frac{\partial P_{e 1}}{\partial P_{2}} & \cdots & \frac{\partial P_{e 1}}{\partial P_{n}} \\
\frac{\partial P_{e 2}}{\partial P_{1}} & \frac{\partial P_{e 2}}{\partial P_{2}} & \cdots & \frac{\partial P_{e 2}}{\partial P_{n}} \\
\frac{\partial P_{e n}}{\partial P_{1}} & \frac{\partial P_{e n}}{\partial P_{2}} & \cdots & \frac{\partial P_{e n}}{\partial P_{n}}
\end{array}\right] \cdot\left[\begin{array}{c}
\Delta P_{1} \\
\Delta P_{2} \\
\vdots \\
\Delta P_{n}
\end{array}\right]
$$

The coefficients $\partial P_{e i} / \partial P_{j}$ constitute sensitivity degrees of machine $i$ to the injected power at different buses, and this will be the basis of the strategy control that we propose later.

\subsection{Load Shedding Concept}

Several control strategies of the electrical network quality are qualified as preventive [4], for example, the decision making is offline. Generally, these strategies, already established, evaluate the consequences of some failures on the network, and, consequently, it is a question of envisaging an action plan which can have failures at the time of a random defect. Our new control strategy is curative. It consists in evaluating online the load quantities to be shed by referring to machine sensitivities to the different injected powers.

The intelligent load shedding procedure is based on fuzzy algorithms. As the temporal evolution of generated power is strongly noticeable to the consumed power level fluctuations, intelligent load shedding algorithms established must, on the one hand, evaluate the gap between the generated power by each machine before and after the failure occurrence and then attenuate the variations of this power in relation to a reference value counted before failure occurrence. Considering the topology of the studied network $\left(N_{\text {bus }}\right.$ buses), the number of fuzzy controllers, affected in the control algorithm of every machine, is equal to $N_{\text {bus }}-1$. Therefore, the total fuzzy controller number is $N_{m} \cdot\left(N_{\text {bus }}-1\right)$. Every fuzzy controller treats two inputs: the first one $\varepsilon_{i}$ represents the gap between the generated power value by the machine $i$ before failure appearance, as reference value $P_{e i}^{*}$, and the one generated during the failure phase $P_{e i}$. The second input represents the generated power variation in relation to the injected power at bus $j$ taken among the $N_{\text {bus }}-1$ remaining buses $\left(\partial P_{e i} / \partial P_{j}\right)$. The taken fuzzy controller decision represents the ratio of loads to be shed at a vulnerable bus: this decision is described by a command vector $U_{c(i, j)}$ (Figure 1).

We notice that the load quantity shed contributes effectively to the restoration of a concerned machine steady state and this by means of a predetermined sensitivity degree.

In order to assign more precision to the action brought by the integrated fuzzy controllers [11] and to take into account the maximum number of states susceptible to affect the different treated variables, we defined nine membership functions for the two input variables: NVL (Negative Very Large), NL (Negative Large), NM (Negative Medium), NS (negative small), ZE (zero), PS (positive small), PM (positive medium), PL (positive large), and PVL (positive very large). We formulated, thus, 81 fuzzy rules which 


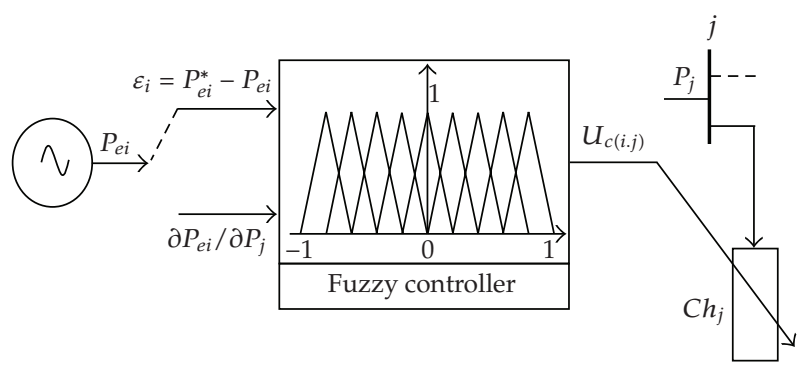

Figure 1: Principle of intelligent load shedding.

Table 1: Different rules of fuzzy decision.

\begin{tabular}{llllllllll}
\hline \multirow{2}{*}{$\varepsilon_{i} / \partial t$} & & & \multicolumn{2}{c}{$\varepsilon_{i}$} & & & & \\
& NVL & NL & NM & NS & ZE & PS & PM & PL & PVL \\
\hline NVL & PVL & PVL & PL & PM & ZE & PS & PL & PL & PL \\
NL & PVL & PVL & PL & PM & ZE & PS & PL & PL & PL \\
NM & PVL & PVL & PL & PM & ZE & PS & PM & PL & PL \\
NS & PVL & PVL & PL & PM & ZE & PS & PM & PL & PL \\
ZE & PVL & PVL & PL & PM & ZE & PS & PM & PL & PL \\
PS & PVL & PL & PM & PS & ZE & PS & PM & PL & PVL \\
PM & PVL & PL & PM & PS & ZE & PS & PM & PL & PVL \\
PL & PVL & PL & PM & PS & ZE & PS & PM & PL & PVL \\
PVL & PVL & PL & PM & PS & ZE & PS & PM & PL & PVL \\
\hline
\end{tabular}

operate the command vector $U_{c(i, j)}$ (Table 1). Membership degrees of variables submitted to the fuzzy controllers are established from Figure 2.

We notice that, as first testing of the fuzzy control reliability integrated in our strategy, we used $25(5 \times 5), 49$ rules $(7 \times 7$ membership functions). In these conditions, we conclude that the decision taken by the controllers (amount of load to be shed) is notably affected and donot satisfy the required stability conditions related to different failures. The more the number of membership functions is important, the more we grant better precision at the moment of the decision making. Thus, in order to insure a refined evaluation for the inputs and outputs of fuzzy controllers ( 2 inputs and one output for each controller) and to cover the different possibility of variables ranging, we adopted 81 rules $(9 \times 9)$ in the fuzzy control strategy. The global strategy of the intelligent load shedding is developed in the next section.

\subsection{Fuzzy Control Adaptation of Studied Model}

Considering the fact that every machine presents a sensitivity degree depending on every load connected to the studied network, the fuzzy controller number $N_{\mathrm{RF}}$ that affected the stability control of every machine is

$$
N_{\mathrm{RF}}=N_{\text {bus }}-1
$$




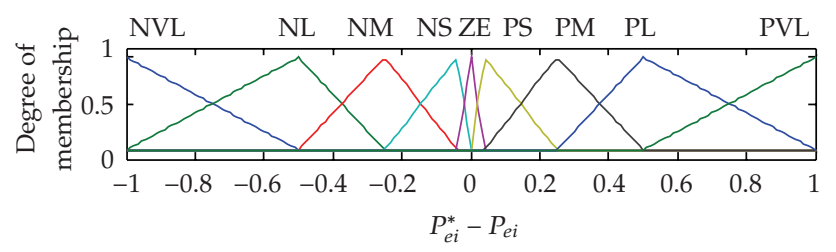

(a)

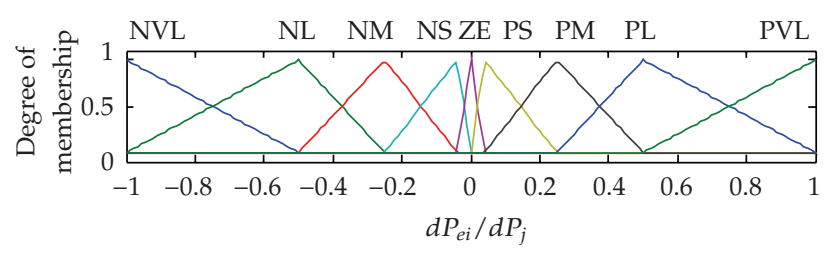

(b)

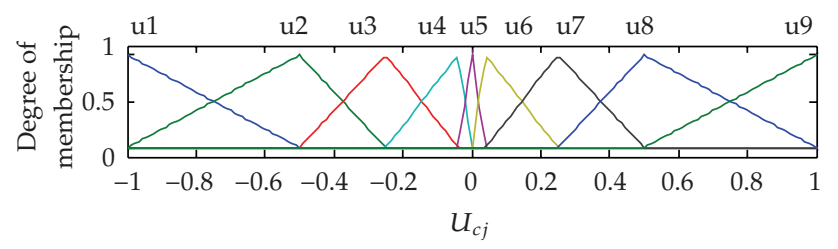

(c)

Figure 2: Membership functions.

Therefore, the total number of fuzzy controllers $N_{\text {TRF }}$ assigned to the studied network topology is

$$
N_{\mathrm{TRF}}=N_{\mathrm{RF}} \cdot N_{m}
$$

where $N_{m}$ is the total machine numbers. We notice that the quantity to be shed at bus $j$ is quantified while taking into account the command vectors $U_{c(i, j)}$ generated by $N_{m}$ machines (Figure 3).

Following the failure initiation, every machine cannot reach a new stability state unless the load shedding ratio is estimated by the fuzzy controllers performed at every load network.

For an intelligent load shedding, the control vector $U_{c j}(n)$ acting on a load $C h_{j}$ is the consequence of vectors $U_{c(k, j)}$, which is expressed as follows:

$$
U_{c j}(n)=\sum_{k=1}^{k=N_{m}} U_{c(k, j)}
$$

Figure 4 represents the reconstitution of the resulting command vector ensuring a load shedding at $C h_{j}$.

The amount of load to be shed is obtained following a decision based on the real demand of power at each bus (sensitivities) that cannot be satisfied by the generators during 


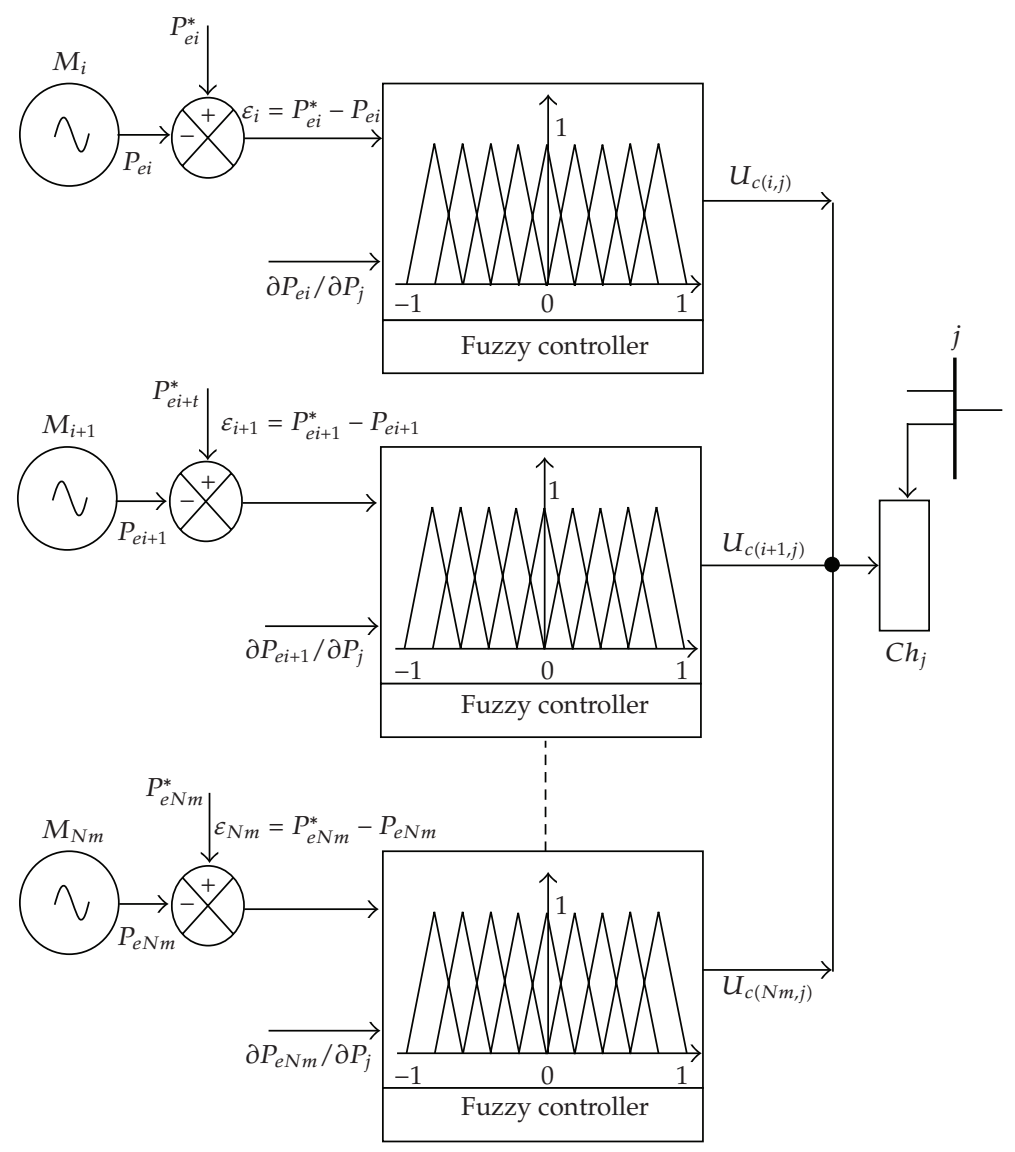

Figure 3: Sensitivity evaluation of different machines to a load.

the failure. This decision is made just at the moment where the fuzzy controllers detect an amount of power generator sensitivity to any injected active power $\partial P_{e i} / \partial P_{j}$. In other words, the formulated decision based on control vector $U_{c(i, j)}$ is not other than the amount of load that must be shed, if not, the voltage level at different buses will be seriously affected. Thus, the whole load shed at bus $j, U_{c j}(n)$ relation (2.10), is perfectly evaluated by the fuzzy controllers.

In order to adapt the variation margins of the studied system electrical variables, $\left(\varepsilon_{i}, \partial P_{i} / \partial P_{j}\right)$ expressed per unit values (p.u.), to variation margins of fuzzy variables, $\left(\varepsilon_{i \text { floue }}, \partial P_{i} /\left.\partial P_{j}\right|_{\text {floue }}\right)$ along the interval $[-1,1]$, we proceeded by an adjustment scale technique by means of a linearized model taking into account the instantaneous controller inputs. The transfer of input variables expressed in the basis p.u. to fuzzy membership domains is done according to the following:

$$
\begin{gathered}
\varepsilon_{i \text { floue }}=a_{\varepsilon i} \cdot \varepsilon_{i}+b_{\varepsilon i} \\
\left.\frac{\partial P_{e i}}{\partial P_{j}}\right|_{\text {floue }}=a_{\Delta \varepsilon i} \cdot \frac{\partial P_{e i}}{\partial P_{j}}+b_{\Delta \varepsilon i} .
\end{gathered}
$$


Under these conditions, the setup scale coefficients are defined by the following parameters recognition:

(i) maximum gaps $\varepsilon_{i \max }$, minimum $\varepsilon_{i \min }$,

(ii) the generated powers,

(iii) variation gaps, $\partial P_{e i} /\left.\partial P_{j}\right|_{\min ^{\prime}} \partial P_{e i} /\left.\partial P_{j}\right|_{\max ^{\prime}}$

(iv) the maximum and minimum consumed power values $P_{c j \max }, P_{c j \text { min }}$.

The quoted maximum and minimum values are reached during the disturbed mode, and they are defined in accordance with

$$
\begin{aligned}
& \varepsilon_{i \max }=P_{e i}^{*}-P_{e i \max }, \\
& \varepsilon_{i \min }=P_{e i}^{*}-P_{e i \min } \\
& \left.\frac{\partial P_{e i}}{\partial P_{j}}\right|_{\max }=C_{p d j \max } \\
& \left.\frac{\partial P_{e i}}{\partial P_{j}}\right|_{\min }=C_{p d j \min }
\end{aligned}
$$

Consequently, the scale adjustment coefficients (2.11) will be

$$
\begin{gathered}
a_{\varepsilon i}=\frac{2}{\left(\varepsilon_{i \max }-\varepsilon_{i \min }\right)}, \\
b_{\varepsilon i}=1-\frac{\left(2 \cdot \varepsilon_{i \max }\right)}{\left(\varepsilon_{i \max }-\varepsilon_{i \min }\right)}, \\
a_{\Delta \varepsilon i}=\frac{2}{\left[\partial P_{e i} /\left.\partial P_{j}\right|_{\max }-\partial P_{e i} /\left.\partial P_{j}\right|_{\min }\right]}, \\
b_{\Delta \varepsilon i}=1-\frac{\left(2 \cdot \Delta \varepsilon_{i \max }\right)}{\left[\partial P_{e i} /\left.\partial P_{j}\right|_{\max }-\partial P_{e i} /\left.\partial P_{j}\right|_{\min }\right]} .
\end{gathered}
$$

The generated control vector by the fuzzy controller is subject to a reverse passage from the fuzzy variable basis to the network basis according to the following linearized model:

$$
U_{c(i, j)}=a_{U_{c(i, j)}} \cdot U_{c(i, j) \text { floue }}+b_{U_{c(i, j)}}
$$

where

$$
\begin{aligned}
& a_{U_{c(i, j)}}=\frac{\left[P_{c j \max }-P_{c j \min }\right]}{2}, \\
& b_{U_{c(i, j)}}=\frac{\left[P_{c j \max }+P_{c j \min }\right]}{2},
\end{aligned}
$$




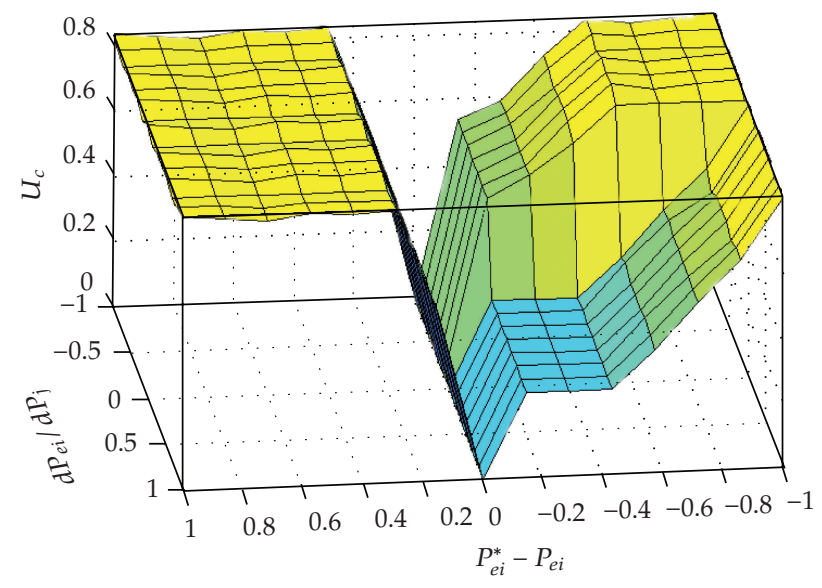

Figure 4: Membership surfaces of vector $U_{c j}(n)$.

$P_{c j \max }$ and $P_{c j \text { min }}$ represent the nominal power and the minimum consumed power by the load, respectively; we remark that the minimum load power value must be fixed in advance while taking into account the socioeconomic constraints. We mention that this scale adjustment technique has been considered power overshoot situations susceptible to appear during failure initiation phases. In fact, the implemented technique possesses dynamic features ensuring some additive performances to the fuzzy controllers (Figure 4).

We present in the next paragraph the established numerical calculation procedure.

\subsection{Calculation Procedure}

We established, under the MATLAB environment, a load flow calculation program of the studied network admitting a dynamic structure. The progress of the calculation respects the steps summarized in Figure 5.

\section{Studied Model}

The studied model is based on the IEEE test of 14 buses network (Figure 6). The dynamic behavior analysis of power system is based on a relevant modeling taking into account the electrodynamics possible states affecting the network in case of serious event appearances. We adopted for the synchronous machines a state model of fourth order as follows:

$$
\begin{gathered}
\frac{d E_{q}^{\prime}}{d t}=\frac{1}{T_{d o}^{\prime}} \cdot\left[E_{f d}-E_{q}^{\prime}+\left(X_{d}-X_{d}^{\prime}\right) \cdot i_{d}\right] \\
\frac{d E_{d}^{\prime}}{d t}=\frac{1}{T_{q o}^{\prime}} \cdot\left[-E_{d}^{\prime}+\left(X_{q}^{\prime}-X_{q}\right) \cdot i_{q}\right] \\
\frac{d \omega}{d t}=\frac{1}{M} \cdot\left[P_{m}-P_{e}-D \cdot(\omega-1)\right] \\
\frac{d \delta}{d t}=\omega-1
\end{gathered}
$$




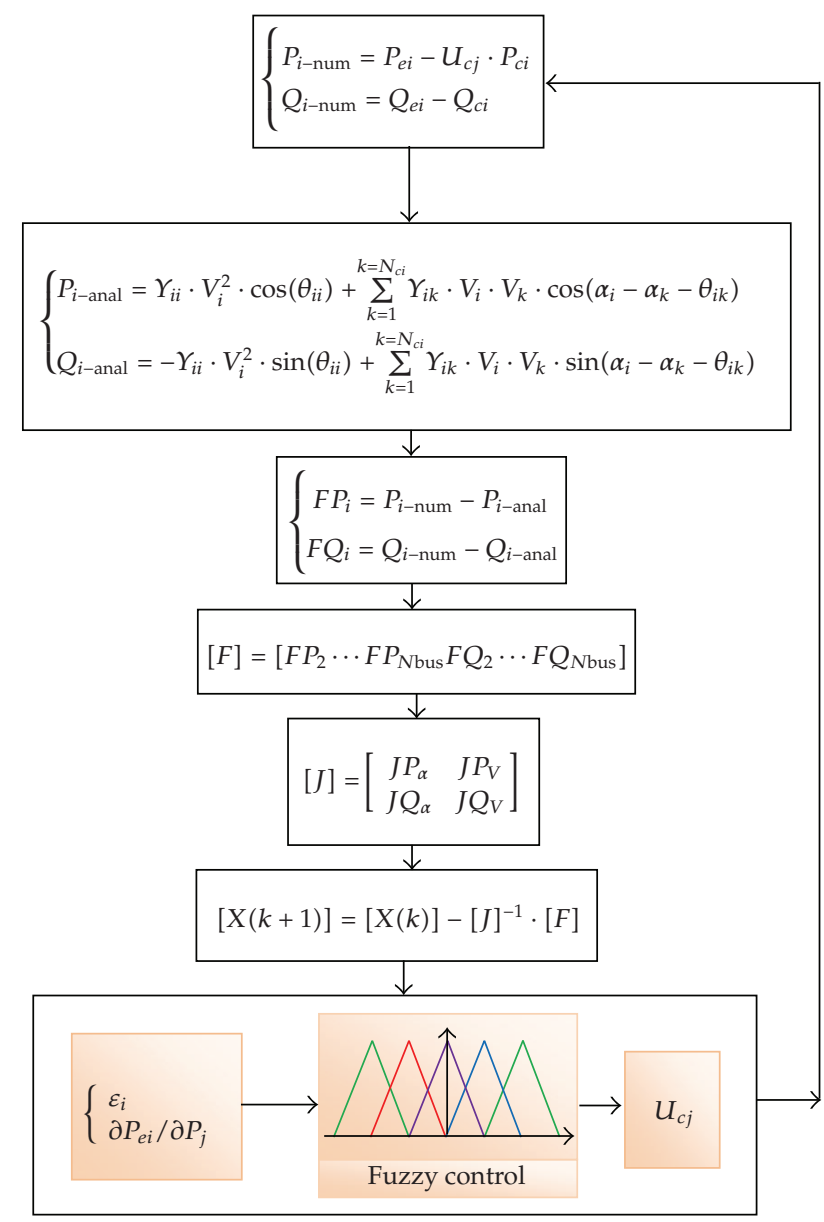

Figure 5: Calculation procedure.

where $E_{d^{\prime}}^{\prime} E_{q}^{\prime}$, and $E_{f d}$ are, respectively, the $(d, q)$ axe transient emf and the emf excitation. $X_{d}, X_{q}, X_{d}^{\prime}$, and $X_{q}^{\prime}$ are, respectively, the $(d, q)$ axe reactances and the $(d, q)$ axe transient reactances. $D$ damping coefficient, $M$ inertia constant. $P_{e}, P_{m}, \omega$, and $\delta$ are, respectively, the electrical power, mechanical power, speed, and the rotor angle.

Each synchronous machine is provided with a speed and voltage regulators [10,12]. The speed regulator $[13,14]$ ensures the frequency control of the generator by an adjustment of the mechanical power delivered to the generator according to the network demand. The voltage regulator $[15,16]$ is based on the detection of the gap between the voltage instantaneous value at machine terminal and a reference value: consequently, we react on the excitation voltage level.

\section{Simulations and Results}

In order to emphasize the contribution of the newly established intelligent control strategy, we simulated three fault scenarios. 


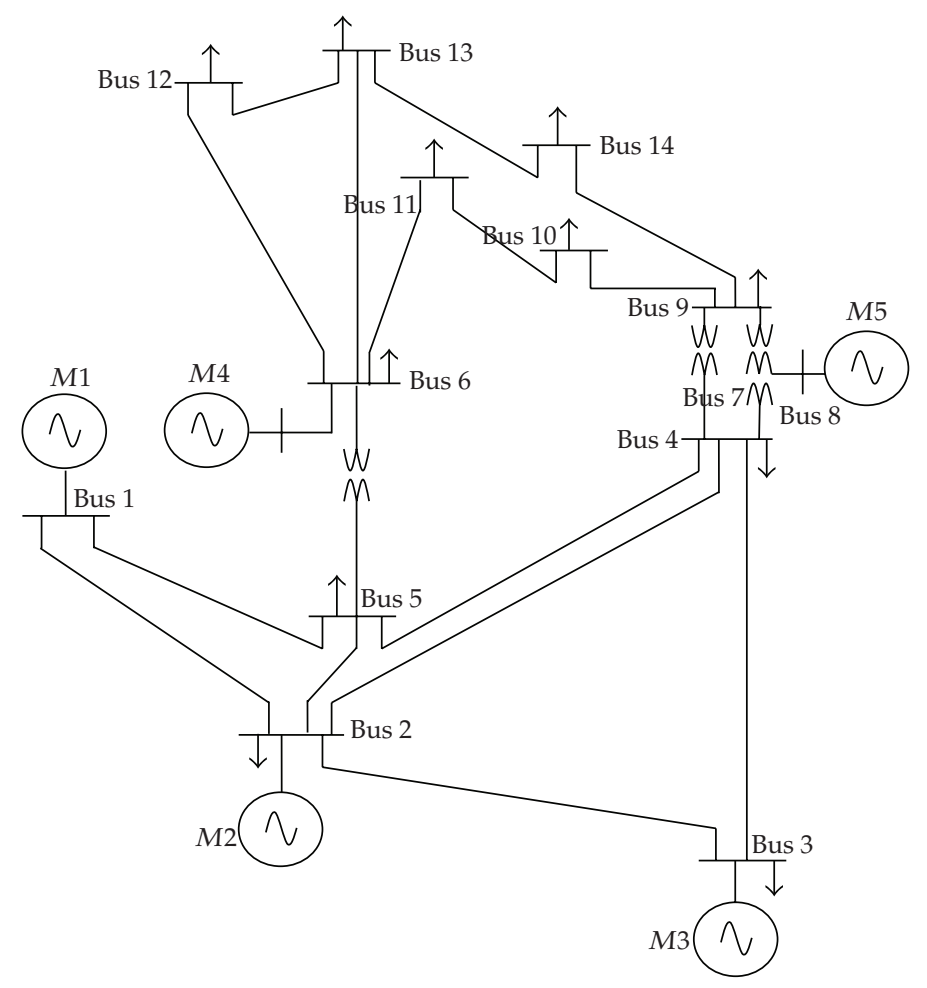

Figure 6: Studied model: IEEE 14-Bus.

First Scenario: Load Increase

At first, we simulated the studied model behavior while adopting the following hypotheses:

(i) absence of load shedding schemes,

(ii) all machines operated with full load except the machine $M_{1}$ (615 MVA) that only delivers 1.16 p.u.

While adopting these assumptions, we supposed that we have at $t=80 \mathrm{~s}$ a new call of load of 4.1 p.u. at bus 2 . Following this, machine 1 reaches its nominal regime. Consequently, the network has no more reserve. This critical situation represents the starting point to check our strategy. Indeed, we considered a total increase of power in all the network of 1.145 p.u. and this at $t=135 \mathrm{~s}$. The consequences of this power increase appeared by a sudden voltage drop at all buses (Figure 7): it is the voltage collapse phenomenon.

Then, we simulated the same scenario while integrating our load shedding strategy. In this case (Figure 8), the establishment of the new load shedding strategy constitutes an efficient remedy to such situations.

Indeed, the fuzzy controllers identified an amount of load to be shed that succeeds in preventing the collapse. This amount of load shed is $0.9452 \mathrm{p} . \mathrm{u}$.

Otherwise, with this controller the voltage level comply with the standards $[4,5]$. Besides, the fuzzy controllers shed a rate of loads reestablishing a steady operating regime according to the sensitivity degree materialized by the generated power level variation depending on the different injected powers (Table 2). The intervention of these controllers 


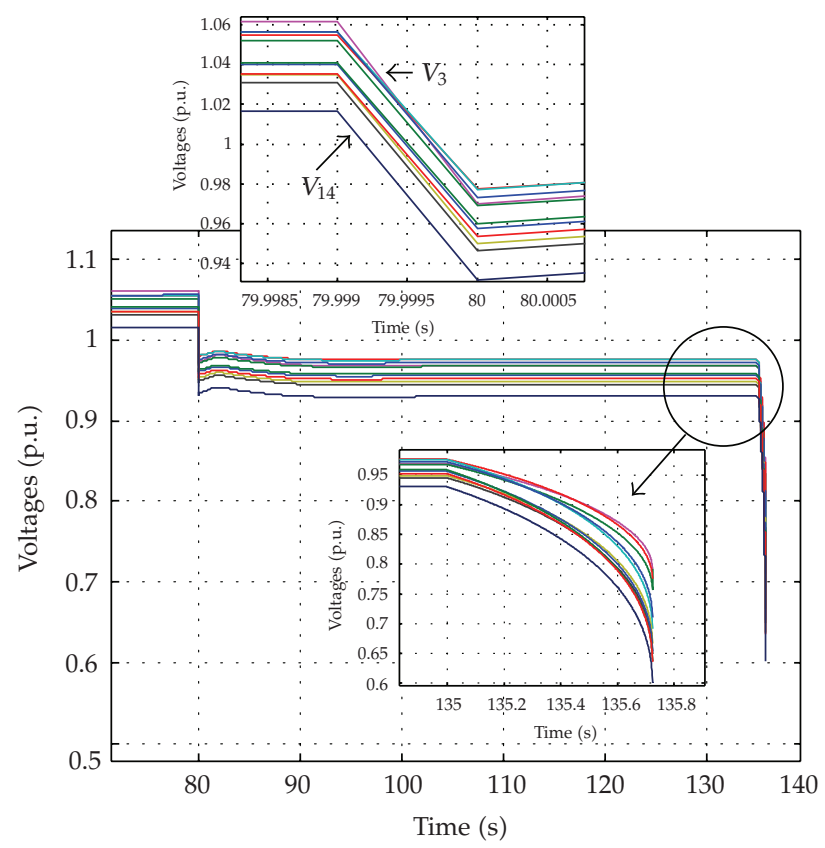

Figure 7: Voltage levels at each bus.

Table 2: Machine sensitivities to injected powers (case of load increase).

\begin{tabular}{lccccc}
\hline & M1 & M2 & M3 & M4 & M5 \\
\hline$\partial P_{e i} / \partial P_{1}$ & 0 & $\mathbf{1 9 3 . 8 0 3}$ & $\mathbf{3 8 . 7 8 0}$ & -3.854 & $-\mathbf{1 5 . 3 4 6}$ \\
$\partial P_{e i} / \partial P_{2}$ & 3.267 & 0 & -3.910 & 0.340 & -0.292 \\
$\partial P_{e i} / \partial P_{3}$ & -1.328 & 0.434 & 0 & -0.077 & 0.109 \\
$\partial P_{e i} / \partial P_{4}$ & -2.330 & 0.296 & -3.910 & -0.206 & 0.241 \\
$\partial P_{e i} / \partial P_{5}$ & 0.953 & 0.010 & $-\mathbf{1 5 . 1 9 0}$ & -0.231 & 0.997 \\
$\partial P_{e i} / \partial P_{6}$ & 0.418 & -0.239 & $-\mathbf{1 3 . 1 4}$ & 0 & -0.355 \\
$\partial P_{e i} / \partial P_{7}$ & $\mathbf{1 9 . 7 3}$ & 1.726 & 2.218 & -2.489 & 2.459 \\
$\partial P_{e i} / \partial P_{8}$ & -2.315 & 1.726 & 2.218 & 0.147 & 0 \\
$\partial P_{e i} / \partial P_{9}$ & $\mathbf{2 . 3 1 5}$ & $\mathbf{0 . 2 9 6}$ & $-\mathbf{0 . 1 6 1}$ & $-\mathbf{0 . 2 0 6}$ & $\mathbf{0 . 2 4 1}$ \\
$\partial P_{e i} / \partial P_{10}$ & 2.330 & -0.296 & 0.161 & 0.206 & -0.241 \\
$\partial P_{e i} / \partial P_{11}$ & -2.147 & 0.367 & 0.035 & -0.153 & 0.212 \\
$\partial P_{e i} / \partial P_{12}$ & -0.242 & 0.167 & 9.01 & 0.315 & 0.244 \\
$\partial P_{e i} / \partial P_{13}$ & -1.012 & 0.672 & 36.927 & -0.029 & 0.998 \\
$\partial P_{e i} / \partial P_{14}$ & 2.066 & -4.808 & -0.338 & 0.124 & -0.195 \\
\hline
\end{tabular}

is fast because voltages took their initial values at the end of $0.012 \mathrm{~s}$ (Figure 8). The fuzzy controller decision is based on machine sensitivity degrees.

Indeed, as shown in Table 2, we notice that machine 2 sensitivity according to the injected power at bus 1 is relatively important compared to those of the other machines. This is due to the fact that machine 2 is connected to the most important load ( $P_{c 2}=4.317$ p.u.), which makes it most sensitive to the disturbance repercussions. Furthermore, some machines (Table 2) possess an important negative sensitivity which are considered as consumers. 


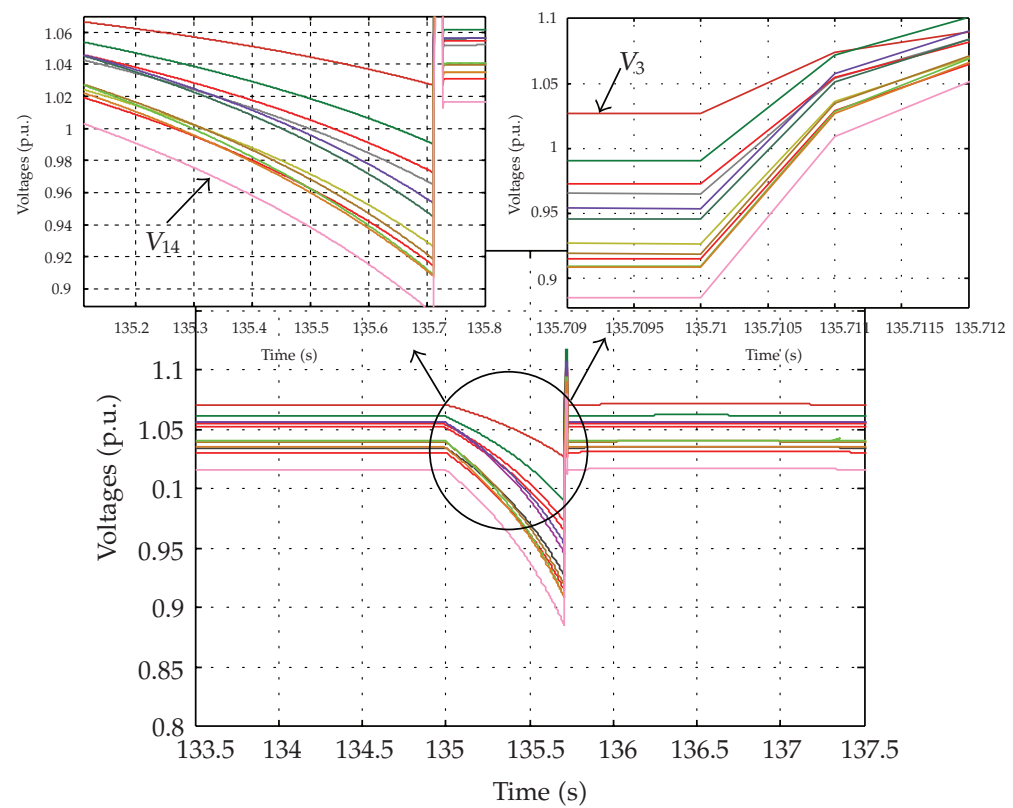

Figure 8: Voltage levels.

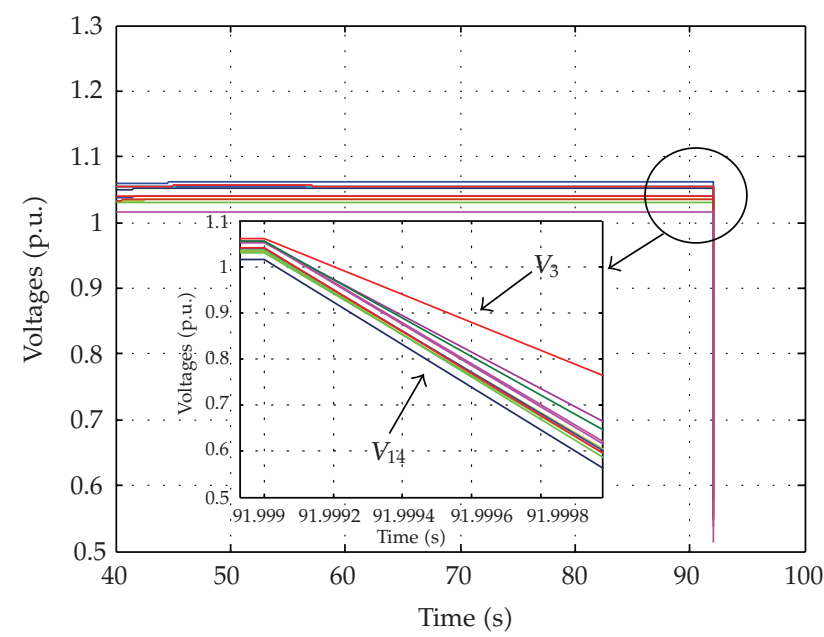

Figure 9: Voltage collapse in case of fuzzy control absence.

\section{Second Scenario: Line Outage}

By this scenario, we supposed that at $t=92 \mathrm{~s}$, the line (1-5) is tripped during $0.01 \mathrm{~s}$. This line (Figure 6) is joined to the most powerful machine and has as an important power flow.

In absence of the intelligent load shedding strategy, the voltage level at different buses undergoes a considerable deterioration leading to a total voltage collapse phenomenon (Figure 9). 


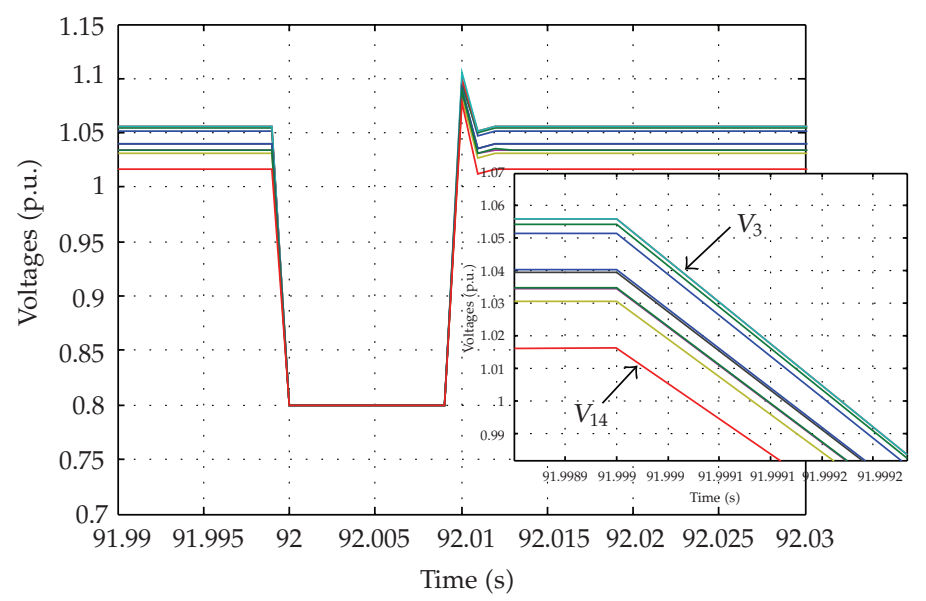

Figure 10: Recuperation of steady voltage levels.

Table 3: Machine sensitivities to injected powers (case of line increase).

\begin{tabular}{|c|c|c|c|c|c|}
\hline & M1 & M2 & M3 & M4 & M5 \\
\hline$\partial P_{e i} / \partial P_{1}$ & 0 & $0.46 \times 10^{6}$ & 2.171 & $3.2 \times 10^{4}$ & $5.6 \times 10^{4}$ \\
\hline$\partial P_{e i} / \partial P_{2}$ & 1.895 & 0 & 2.85 & 0.204 & 0.095 \\
\hline$\partial P_{e i} / \partial P_{3}$ & -1.804 & 0.536 & 0 & -0.006 & 0.102 \\
\hline$\partial P_{e i} / \partial P_{4}$ & -1.321 & 0.136 & 2.85 & -0.115 & 0.189 \\
\hline$\partial P_{e i} / \partial P_{5}$ & 0.618 & -0.072 & 0.443 & -0.102 & -0.625 \\
\hline$\partial P_{e i} / \partial P_{6}$ & -0.01 & 0.992 & 0.055 & 0 & -0.072 \\
\hline$\partial P_{e i} / \partial P_{7}$ & 37.13 & 0.992 & 1.174 & -0.067 & 1.132 \\
\hline$\partial P_{e i} / \partial P_{8}$ & -4.08 & 0.464 & 1.174 & 0.006 & 0 \\
\hline$\partial P_{e i} / \partial P_{9}$ & 4.08 & -0.464 & 0.09 & -0.115 & 0.182 \\
\hline$\partial P_{e i} / \partial P_{10}$ & 1.33 & 0.451 & -0.09 & 0.115 & -0.181 \\
\hline$\partial P_{e i} / \partial P_{11}$ & -1.363 & 0.968 & 0.04 & -0.107 & 0.176 \\
\hline$\partial P_{e i} / \partial P_{12}$ & -0.035 & 0.063 & -0.058 & 0.172 & 0.063 \\
\hline$\partial P_{e i} / \partial P_{13}$ & -0.105 & 0.197 & -0.175 & 0.097 & 0.213 \\
\hline$\partial P_{e i} / \partial P_{14}$ & 2. 03 & -3.586 & -0.321 & 0.090 & -0.017 \\
\hline
\end{tabular}

However, when we integrated our load shedding strategy, we notice again that the fuzzy controllers that appropriately estimated the load quantity to be shed was 1.9795 p.u. This led the voltages to regain an admissible level by norms (0.8 p.u.) (Figure 10).

The tripping of the line (1-5) affected remarkably all machines (Table 3), particularly machine $2\left(\partial P_{e 2} / \partial P_{1}=0,45 \cdot 10^{6}\right)$; this is explained by the fact that the line (1-2) (Figure 1) ensures, in these conditions, a supplementary power flow.

\section{Third Scenario: Generator Outage}

We granted a particular focus on the electrical network behavior in case of one generator outage, when they were operating under nominal conditions (full load). For our studied model, we put machine 1 which was the most powerful out of service using our new strategy. 


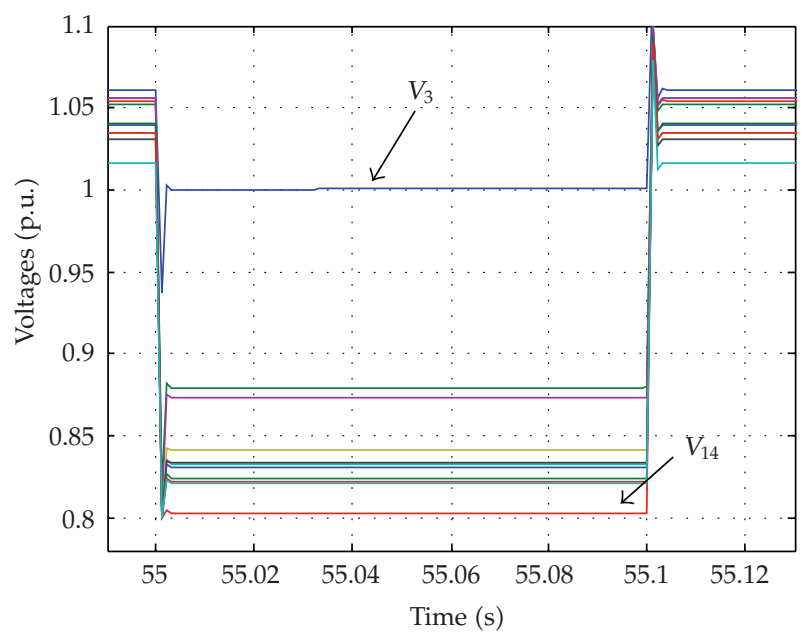

Figure 11: Voltage evolutions.

Table 4: Machine sensitivities to injected powers (case of generator outage).

\begin{tabular}{lccccc}
\hline & M1 & M2 & M3 & M4 & M5 \\
\hline$\partial P_{e i} / \partial P_{1}$ & 0 & $-\mathbf{1 4 . 7 0 8}$ & 1.884 & 0.227 & 1.701 \\
$\partial P_{e i} / \partial P_{2}$ & 0 & 0 & $\mathbf{1 8 . 9 9 7}$ & 0.093 & -0.171 \\
$\partial P_{e i} / \partial P_{3}$ & 0 & 0.779 & 0 & 0.043 & 0.102 \\
$\partial P_{e i} / \partial P_{4}$ & 0 & 1.012 & $\mathbf{1 8 . 9 9 7}$ & -0.070 & 0.144 \\
$\partial P_{e i} / \partial P_{5}$ & 0 & 0.344 & 0.519 & -0.065 & 0.470 \\
$\partial P_{e i} / \partial P_{6}$ & 0 & -0.064 & -0.107 & 0 & -0.027 \\
$\partial P_{e i} / \partial P_{7}$ & 0 & 1.197 & 1.314 & -0.288 & 1.934 \\
$\partial P_{e i} / \partial P_{8}$ & 0 & 1.197 & 1.314 & 0.022 & 0 \\
$\partial P_{e i} / \partial P_{9}$ & 0 & 1.012 & -1.055 & -0.070 & 0.145 \\
$\partial P_{e i} / \partial P_{10}$ & 0 & -1.012 & 1.055 & 0.070 & -0.145 \\
$\partial P_{e i} / \partial P_{11}$ & 0 & 0.976 & 0.014 & -0.067 & 0.138 \\
$\partial P_{e i} / \partial P_{12}$ & 0 & 0.059 & 0.097 & 0.989 & 0.024 \\
$\partial P_{e i} / \partial P_{13}$ & 0 & 0.176 & 0.294 & 2.826 & 0.074 \\
$\partial P_{e i} / \partial P_{14}$ & 0 & -3.097 & -1.035 & 0.062 & -0.139 \\
\hline
\end{tabular}

During the fault phase, machines 2 and 3 contribute to compensate the resulting lack of power. The fuzzy controllers proceeded by a load shedding rate: 1.3308 p.u., that has been given by machine 1 taking into account capacities of the operational machines. The intelligent load shedding strategy imposed a steady operating regime to different machines during the fault phase. We recorded a voltage level variation at different buses (Figure 11) without violating the admissible limits given by norms.

Sensitivity values evaluated for different machines according to injected powers at different buses (Table 4) justify that machine 2 underwent considerably the fault impact. Yet, machine 3 resisted against this disturbance because its sensitivity degree is positive.

As a perspective of performance analysis of the implemented strategy as well as its adaptability with the vulnerable situations met by the studied network, we paid an important attention for a comparative study of the initiated action by the fuzzy controllers at every bus 


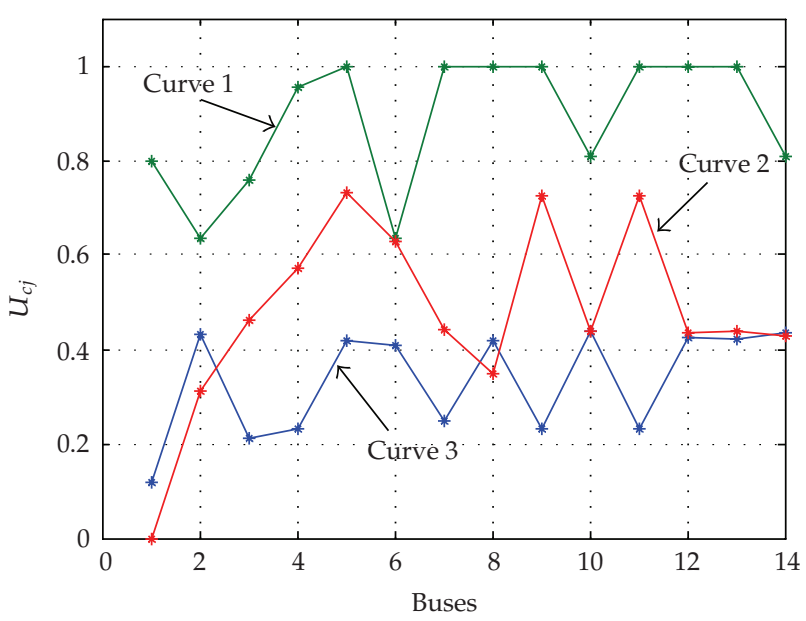

Figure 12: Sensitivity evolution at different buses.

according to some initiated faults (Figure 12) as the line outage (1-5) (curve 1), generator 1 outage (curve 2), and the load increase (curve 3). We noticed that the fuzzy controllers shed, during every fault phase, a total load quantity which no machine can provide. Therefore, this new intelligent load shedding strategy guaranteed new steady states of power balance. We have

$$
\sum_{i=1}^{i=N m} P_{e i}=\sum_{k=1}^{k=N_{\text {bus }}}\left(1-U_{c k}\right) \cdot P_{c k}+\text { losses. }
$$

\section{Conclusion}

The major objective aimed by our paper is to ensure the network service continuity in presence of vulnerable situations. This strategy has been founded, on the one hand, on perfect sensitivity degree recognition of every machine relating to injected power levels at different buses and, on the other, on the generated power level variation by every machine, in relation to a reference value evaluated in normal operating regime. Various fault scenarios with serious characters have been initiated in different network zones in order to inquire, primarily, the fault seriousness and, then, to test the implemented strategy potentialities. The actions of many fuzzy controllers hired in this strategy have not been corrective without an appropriated development of the studied electrical network state models while granting a particular importance to the machine behaviors under disrupted mode. Versatile simulations have been done according to numerical model establishments taking into account the instantaneous power exchanges between machines and network. These models have been endowed with a dynamic structure allowing them an adjusted adaptability with topologies specifically acquired by the network during disturbed mode. A deep analysis of the obtained results, following various simulations, shows our newly strategy potentialities.

\section{Acknowledgment}

The authors are deeply grateful to the anonymous referees for careful reading of this paper and constructive comments. 


\section{References}

[1] S. Chebbi, H. Jouini, and M. Annabi, "Application of SIME method to tunisian network," in Proceedings of the International Conference on Electric Power and Energy Conversion Systems (EPECS '09), IEEE, November 2009.

[2] R. Faranda, A. Pievatolo, and E. Tironi, "Load shedding: a new proposal," IEEE Transactions on Power Systems, vol. 22, no. 4, pp. 2086-2093, 2007.

[3] D. K. Subramanian, "Optimum load shedding through programming techniques," IEEE Transactions on Power Apparatus and Systems, vol. 90, no. 1, pp. 89-95, 1971.

[4] T. Tomšič, G. Verbič, and F. Gubina, "Revision of the underfrequency load-shedding scheme of the Slovenian power system," in Proceedings of the IEEE Power Engineering Society General Meeting, pp. 1782-1787, June 2005.

[5] C. J. Parker, I. F. Morrison, and D. Sutanto, "Simulation of load shedding as a corrective action against voltage collapse," Electric Power Systems Research, vol. 46, no. 3, pp. 235-241, 1998.

[6] A. M.A. Haidar, A. Mohamed, and A. Hussain, "Vulnerability control of large scale interconnected power system using neuro-fuzzy load shedding approach," Expert Systems with Applications, vol. 37, no. 4, pp. 3171-3176, 2010.

[7] A. A. Girgis and S. Mathure, "Application of active power sensitivity to frequency and voltage variations on load shedding," Electric Power Systems Research, vol. 80, no. 3, pp. 306-310, 2010.

[8] A. N. Udupa, G. K. Purushothama, K. Parthasarathy, and D. Thukaram, "Fuzzy control for network overload alleviation," International Journal of Electrical Power and Energy System, vol. 23, no. 2, pp. 119 $128,2001$.

[9] S. J. Huang and C. C. Huang, "Adaptive load shedding method with time-based design for isolated power systems," International Journal of Electrical Power and Energy System, vol. 22, no. 1, pp. 51-58, 2000.

[10] H. Jouini, S. chebbi, and M. Annabi, "Technique of unballasting of load in an electrical network supply," in Proceedings of the International Review on Modelling and Simulations (IREMOS '08), vol. 1, October 2008.

[11] IEEE Standards Board, IEEE Recommended Practice for Excitation System Models for Power System Stability Studies, Institute of Electrical and Electronics Engineers, New York, NY, USA, 1992.

[12] P. Kundur, Power System Stability and Control, Electric Power Research Institute,Power System Engineering Series, McGraw-Hill, New York, NY, USA, 1998.

[13] P. M. Anderson and A. A. Foued, Power System Stability and Control, IEEE Press Power Systems Engeneering, The institute of Electrical and Electronics Engineers, New York, NY, USA, 1993.

[14] L. N. Hannett, J. W. Feltes, and B. Fardanesh, "Field tests to validate hydro turbine-governor model structure and parameters," IEEE Transactions on Power Systems, vol. 9, no. 4, pp. 1744-1751, 1994.

[15] Working group on prime mover and Energy Supply Models for system dynamic performances studies, "Hydraulic turbine and turbine control models for system dynamic studies," IEEE Transactions on Power Systems, vol. 7, no. 1, 1992.

[16] L. M. Hajagos and M. J. Basler, “Changes to IEEE 421.5 recommended practice for excitation system models for power system stability studies," in Proceedings of the IEEE Power Engineering Society General Meeting (PES '05), vol. 1, pp. 974-976, San Francisco, Calif, USA, 2005. 


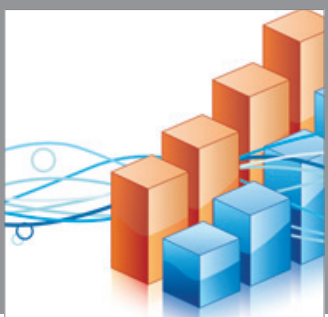

Advances in

Operations Research

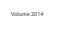

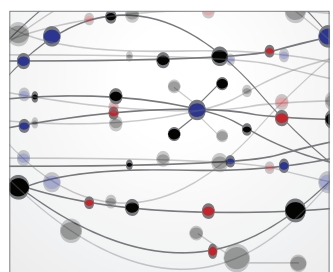

\section{The Scientific} World Journal
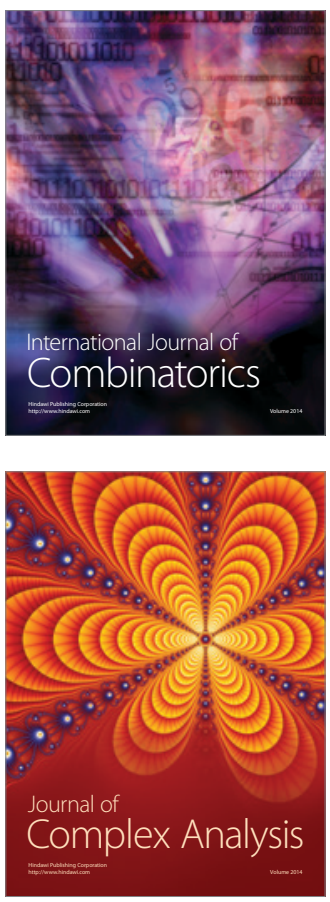

International Journal of

Mathematics and

Mathematical

Sciences
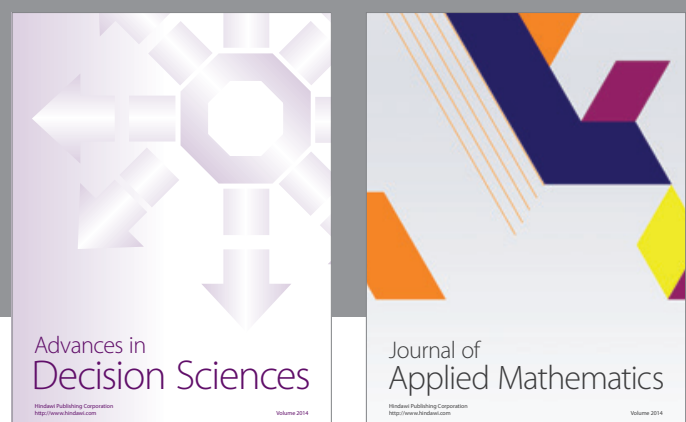

Journal of

Applied Mathematics
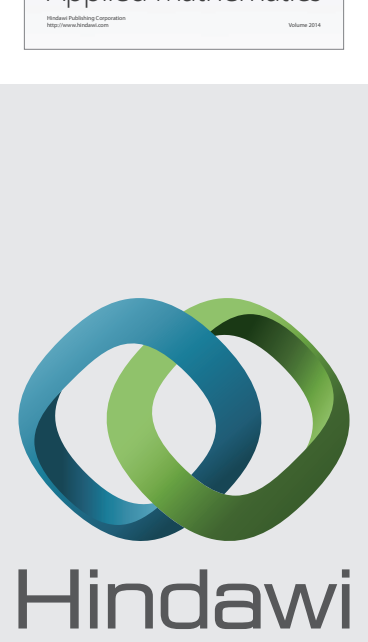

Submit your manuscripts at http://www.hindawi.com
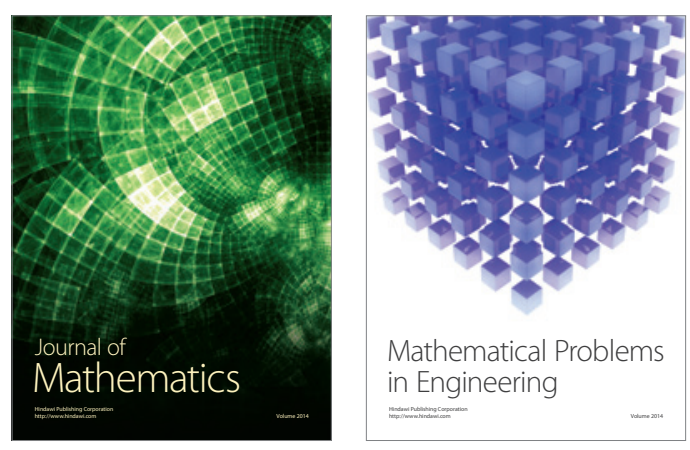

Mathematical Problems in Engineering
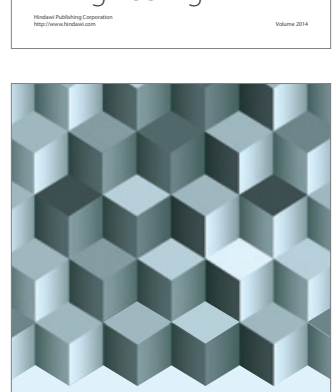

Journal of

Function Spaces
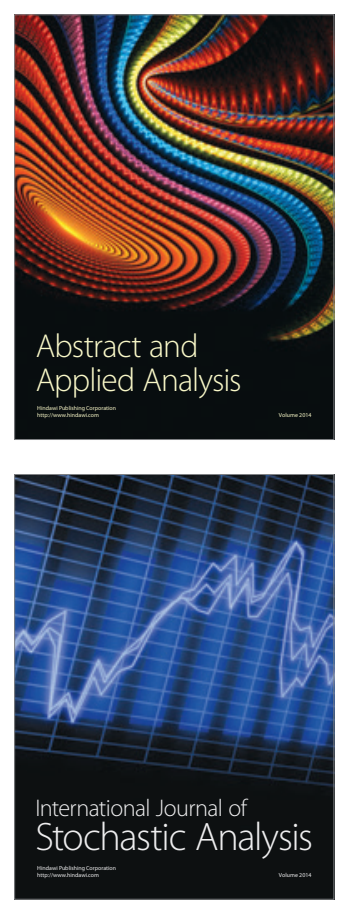

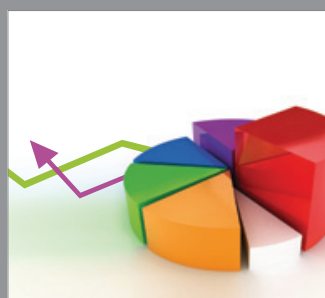

ournal of

Probability and Statistics

Promensencen
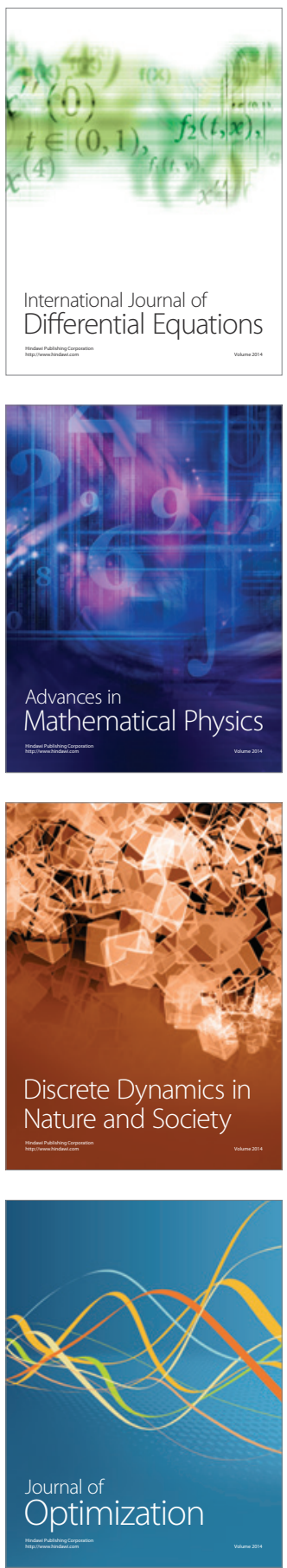